Pacific Journal of Mathematics

THEORY OF BOUNDED GROUPS AND THEIR BOUNDED 


\title{
THEORY OF BOUNDED GROUPS AND THEIR BOUNDED COHOMOLOGY
}

\author{
D. W. PAUl
}

\begin{abstract}
Bounded cohomology $H_{b}^{*}$ can be defined for groups and for topological spaces. Recent work has shown that $H_{b}^{*}(M)$ of a topological space $M$ depends only on $\Pi_{1}(M)$. In this paper we consider a new concept-a bounded group-and thereby expand the definition of bounded cohomology. We prove that bounded cohomology groups are themselves bounded groups and develop their properties in lower dimensions. In particular, elements of $H_{b}^{2}(G, A)$ classify bounded group extensions of $G$ by $A$. As an application of the theory of bounded groups we construct the Lyndon spectral sequence. The result obtained is Theorem 3 , which states that $H_{b}^{n}(H, A)^{G} \cong_{b} H_{b}^{n}(G, A)$, when $G / H$ is finite.
\end{abstract}

1. Definitions and observations. We introduce the ideas of a bounded group and a bounded group homomorphism. We want to ensure that the mappings $(x, y) \rightarrow x y$ and $x \rightarrow x^{-1}$ are themselves bounded homomorphisms. Thus, we define a norm (actually a pseudo-norm) on a group $G$ to be a function \|\|$: G \rightarrow R$ satisfying, for non-negative constants $M, c, M^{\prime}, c^{\prime}$,

(i) $\|x\| \geq 0$, for all $x$ in $G$,

(ii) $\|x y\| \leq M(\|x\|+\|y\|)+c$,

(iii) $\left\|x^{-1}\right\| \leq M^{\prime}\|x\|+c^{\prime}$.

$G$ together with its norm is a bounded group. A homomorphism $f$ between two bounded groups $G$ and $H$ is bounded if and only if there exist non-negative constants $M_{f}$ and $c_{f}$ such that

$$
\|f(g)\| \leq M_{f}\|g\|+c_{f}, \quad \text { for all } g \text { in } G .
$$

Two norms on $G$ are equivalent if there exists a bounded isomorphism $f$ between the two bounded groups; i.e., both $f$ and $f^{-1}$ must be bounded. The symbol for a bounded isomorphism will be $\cong_{b}$. It is worth noting that the composition of two bounded homomorphisms is bounded.

The cross-product of two bounded groups, $G \times H$, is a bounded group under the natural norm defined as follows:

$$
\|(g, h)\|=\|g\|+\|h\| \text {. }
$$


Here, \|\| is the symbol for the norm in the appropriate group. Notice that the choices of definitions for bounded group, bounded homomorphism, and cross-product norm achieve the stated goal of making $\left(g_{1}, g_{2}\right) \rightarrow g_{1} g_{2}$ and $g \rightarrow g^{-1}$ bounded homomorphisms.

The need for a pseudo-norm rather than a norm arises as we attempt to define a quotient norm for $G / H$, when $H$ is normal in $G$. Given $\bar{g}$ in $G / H$, define

$$
\|\bar{g}\|=\inf _{h \in H}\|g h\| .
$$

This norm satisfies properties (i)-(iii); however, the condition $\|\bar{g}\|=0$ does not imply that $g$ is in $H$. Notice that the projection map $\pi: G \rightarrow$ $G / H$ is a bounded homomorphism.

The quotient norm will appear repeatedly in this work, so we prove now a simple and crucial lemma.

LEMMA 1. Let $H$ be a normal subgroup of bounded group $G$, and let $\Pi: G \rightarrow G / H$ be the projection map. Endow $G / H$ with the quotient norm. Then there exists a bounded section $s: G / H \rightarrow G$, so that $\Pi \circ s=$ 1.

Proof.

$$
\|\Pi(g)\|=\inf _{h \in H}\|g h\|=L .
$$

Given $\varepsilon>0$, for each $g$ there exists an $h_{\varepsilon}$ in $H$ such that

$$
\left\|g h_{\varepsilon}\right\| \leq L+\varepsilon \text {. }
$$

Pick such an $h_{\varepsilon}$, and define

$$
s(\Pi(g))=g h_{\varepsilon}
$$

Then $\|s(\Pi(g))\|=\left\|g h_{\varepsilon}\right\| \leq L+\varepsilon=\|\Pi(g)\|+\varepsilon$.

The bounded cohomology groups of a bounded group $G$ are defined directly in terms of the bar resolution [2]. Let $A$ and $G$ be bounded groups, and let $A$ be a left $G$-module. The $G$-module action must be bounded; i.e.,

$$
\|g a\| \leq M\|a\|+c \text {. }
$$

The chain complex consists of free $G$-modules $B_{n}(G)$. In non-homogeneous coordinates, $B_{n}(G)$ has generators $\left[x_{1} / \cdots / x_{n}\right]$, n-tuples of elements of $G$, with the normalization condition that

$$
\left[x_{1} / \cdots / x_{n}\right]=0 \quad \text { if any } x_{i}=1 .
$$


The group $B_{n}(G)$ is bounded with the following norm inherited from $G$ :

$$
\left\|x\left[x_{1} / \cdots / x_{n}\right]\right\|=\|x\|+\left\|x_{1}\right\|+\cdots+\left\|x_{n}\right\| .
$$

For $a$ and $b$ in $B_{n}(G),\|a+b\|=\|a\|+\|b\|$. An $n$-cochain $f: B_{n}(G) \rightarrow A$ is a $G$-module homomorphism, bounded as a group homomorphism, uniquely determined by its values on the generators. These $n$-cochains form a group and are denoted $\operatorname{Hom}_{G}^{b}\left(B_{n}(G), A\right)$, or $B^{n}$. Because $A$ has bounded $G$-module action, $\delta f$ is also a bounded cochain, and thus it is in $B^{n+1}$. The cohomology groups $H_{b}^{n}(G, A)$ are those of the cochain complex $\operatorname{Hom}_{G}^{b}\left(B_{n}(G), A\right)$.

The bar resolution is also defined in homogeneous coordinates. In this case, $B_{n}^{\prime}(G)$ consists of all elements $\left(x_{0}, \ldots, x_{n}\right)$, where $\left(x_{0}, \ldots, x_{n}\right)$ $=0$ when $x_{i}=x_{i+1}$. We define the norm of elements of $B_{n}^{\prime}(G)$ by the formula

$$
\left\|\left(x_{0}, \ldots, x_{n}\right)\right\|=\left\|x_{0}\right\|+\cdots+\left\|x_{n}\right\| .
$$

LEMMA 2 . $B_{n}(G) \cong_{b} B_{n}^{\prime}(G)$.

Proof. The two resolutions are algebraically isomorphic [2]; an element $x\left[x_{1} / \cdots / x_{n}\right]$ in $B_{n}(G)$ determines one in $B_{n}^{\prime}(G)$ by

$$
x\left[x_{1} / \cdots / x_{n}\right] \rightarrow\left(x, x x_{1}, \ldots, x x_{1} \cdots x_{n}\right) .
$$

Conversely,

$$
\left(y_{0}, \ldots, y_{n}\right) \rightarrow y_{0}\left[y_{0}^{-1} y_{1} / \cdots / y_{n-1}^{-1} y_{n}\right] .
$$

For the proof of boundedness, note that in each direction elements of $G$ are multiplied a finite number of times before the cross-product norm is applied. For a fixed $n$, these actions are bounded, and thus $B_{n}(G)$ and $B_{n}^{\prime}(G)$ are bounded isomorphic.

We would like to know if the quotient $H_{b}^{n}(G, A)$ itself can be considered bounded. First, a natural norm to assign an $n$-cocycle $f$ in $\operatorname{Hom}_{G}^{b}\left(B_{n}(G), A\right)$ is the sup norm.

$$
\|f\|=\sup _{\|x\| \leq 1}\|f(x)\| .
$$

Verifying the conditions for $Z_{b}^{n}(G, A)$ (the $n$-cocycles) to be a bounded group depends on knowing that $f$ is bounded and that $A$ is a bounded group. Now we define the norm for the cohomology class of $f$ by the quotient norm:

$$
\|\bar{f}\|=\inf _{\delta g \in B^{n}}\|f+\delta g\|
$$


Now the cohomology groups $H_{b}^{n}(G, A)=Z_{b}^{n}(G, A) / B_{b}^{n}(G, A)$ are themselves bounded groups.

2. Low dimensional cohomology groups. We first note two standard results about the zero- and one-dimensional bounded cohomology groups. Given a bounded $G$-module action on any bounded group $A$, it is clear that $H_{b}^{0}(G, A)$ is $A^{G} . H_{b}^{1}(G, A)$ is easily seen to be isomorphic to the group of all bounded crossed homomorphisms modulo the principal ones. Our concern is with the relationship between $H_{b}^{2}(G, A)$ and extensions. We develop the theory for the case of central extensions and trivial $G$-module action. The results easily extend to the case of bounded module action.

Let $G$ and $K$ be bounded groups, and let $K$ be abelian. A bounded central extension $E$ of $K$ by $G$ is defined as a central extension with bounded homomorphisms $i$ and $\pi$.

$$
E: 0 \longrightarrow K \stackrel{i}{\longrightarrow} X \stackrel{\pi}{\longrightarrow} G \longrightarrow 1
$$

Let $E$ and $E^{\prime}$ be two bounded central extensions of $K$ by $G$; then $E$ and $E^{\prime}$ are congruent if there exists a bounded homomorphism $\beta$, with bounded inverse $\beta^{-1}$, such that the following diagram commutes:

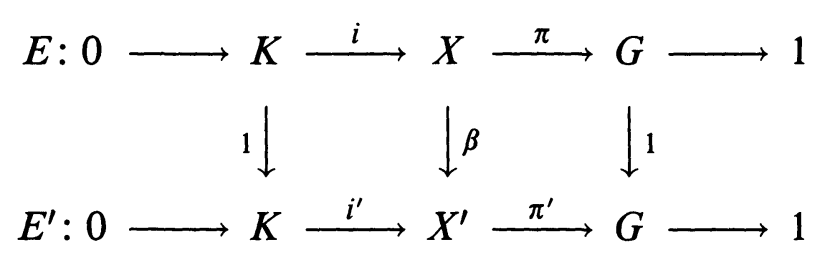

The collection of such congruence classes is denoted $\operatorname{Cent}_{b}(G, K)$.

Assuming non-homogeneous coordinates and trivial $G$-module action on $K$, we construct the groups $H_{b}^{2}(G, K)$. An $n$-cocycle $f$ has the property that

$$
\left\|f\left(x_{1}, \ldots, x_{n}\right)\right\| \leq M \|\left(\left\|x_{1}\right\|+\cdots+\left\|x_{n}\right\|\right)+c .
$$

Our goal is to associate a congruence class in $\operatorname{Cent}_{b}(G, K)$ to each cohomology class in $H_{b}^{2}(G, K)$.

Given $f$ in $H_{b}^{2}(G, K)$ as just defined, form the group $X$ consisting of all pairs $(k, g), k$ in $K$ and $g$ in $G$. The group operation is defined

$$
\left(k_{1}, g_{1}\right)\left(k_{2}, g_{2}\right)=\left(k_{1}+k_{2}+f\left(g_{1}, g_{2}\right), g_{1} g_{2}\right) \text {. }
$$

A bounded group was defined in order to make this extension group $X$ bounded. 
LEMMA 3. $X$ is a bounded group under the following norm:

$$
\|(k, g)\|=\|k\|+\|g\| \text {. }
$$

Proof. Clearly $\|(k, g)\| \geq 0$.

$$
\left\|\left(k_{1}, g_{1}\right)\left(k_{2}, g_{2}\right)\right\|=\|\left(k_{1}+k_{2}+f\left(g_{1}, g_{2}\right), g_{1} g_{2}\right) \text {. }
$$

The bounds from the group operations in $K$ and $G$ combine with the bound on $f$ to make the operation in $X$ bounded. Similarly, we see that $\|(k, g)\| \rightarrow\left\|(k, g)^{-1}\right\|=\left\|\left(-k-f\left(g, g^{-1}\right), g^{-1}\right)\right\|$ is bounded.

The homomorphisms are defined to be:

$$
\begin{array}{ll}
i: K \rightarrow X, & i(k)=(k, 1), \\
\pi: X \rightarrow G, & \pi(k, g)=g .
\end{array}
$$

LEMMA 4. The homomorphisms $i$ and $\pi$ are bounded.

Proof.

$$
\begin{gathered}
\|i(k)\|=\|(k, 1)\|=\|k\|+c . \\
\|\pi(k, g)\|=\|g\| \leq\|k\|+\|g\| .
\end{gathered}
$$

LEMMA $5 . X / K \cong_{b} G$.

Proof. Let $\left(\overline{k, g_{0}}\right)$ represent a class in $X / K$.

The groups are known to be isomorphic; the boundedness of each direction of the isomorphism is demonstrated here.

$$
\begin{gathered}
\left\|\left(\overline{k, g_{0}}\right)\right\|=\inf _{k}\left\|\left(k, g_{0}\right)\right\|=\inf _{k}\|k\|+\left\|g_{0}\right\|=\left\|g_{0}\right\|+c . \\
\left\|g_{0}\right\| \leq \inf _{k}\|k\|+\left\|g_{0}\right\| \leq\left(\overline{k, g_{0}}\right) .
\end{gathered}
$$

The proofs of the exactness of the sequence

$$
E: 0 \longrightarrow K \stackrel{i}{\longrightarrow} X \stackrel{\pi}{\longrightarrow} G \longrightarrow 1
$$

and of $i(K)$ lying in the center of $X$ are standard [2].

Thus we have easily found a bounded central extension for the twococycle $f$. 
LEMMA 6. If $f$ and $g$ are cohomologous, then their associated extensions $X$ and $X^{\prime}$ are congruent.

Proof. Let $g=f+\delta d$, and note that $d$ must be bounded. Define $\beta(k, g)=(k-d(g), g) ; d$ is bounded and thus $\beta$ is a bounded homomorphism. The inverse $\beta^{-1}$, defined $\beta^{-1}(k, g)=(k+d(g), g)$, is also bounded. The map $\beta$ makes the diagram commute.

Given a bounded central extension $E$, we now wish to find an associated two-cocycle in $Z_{b}^{2}(G, K)$.

$$
E: 0 \longrightarrow K \stackrel{i}{\longrightarrow} \underset{k-s^{-}}{\stackrel{\pi}{\longrightarrow}} G \longrightarrow 1 .
$$

Since $G$ has a norm equivalent to the quotient norm, there exists a bounded section $s: G \rightarrow X$ by Lemma 1 . We require further that $s(1)=1$, which may only affect the bounded condition of $s$ by a constant. Define $\Delta$ in $Z_{b}^{2}(G, K)$ by

$$
\Delta(a, b)=s(a) s(b) s(a b)^{-1}
$$

The class of $\Delta$ is easily seen to be an element of $Z^{2}(G, K)$; as a composition of a finite number of group operations it is bounded.

LEMMA 7. If $E$ and $E^{\prime}$ are congruent bounded central extensions

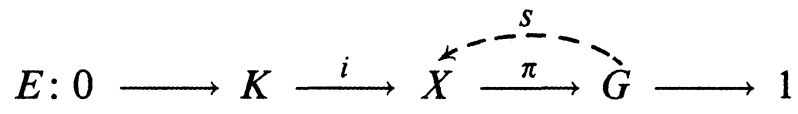

$$
\begin{aligned}
& 1 \downarrow \downarrow \beta 1 \\
& E^{\prime}: 0 \longrightarrow K \stackrel{i^{\prime}}{\longrightarrow} \underset{X_{-}^{\prime}}{\stackrel{\pi^{\prime}}{\longrightarrow} G \longrightarrow} \longrightarrow
\end{aligned}
$$

with bounded sections $s$ and $t$, then their associated cocycles $\Delta_{s}$ and $\Delta_{t}$ are cohomologous as bounded cocycles.

Proof. Define $d: B_{1}(G) \rightarrow K$ by

$$
d(g)=t(g) \beta(s(g))^{-1}
$$

Standard arguments show that $\Delta_{t}=\Delta_{s}+\delta d$. Since $d$ is bounded as a composition of $\beta, t$, and $s, \Delta_{t}$ and $\Delta_{s}$ are in the same bounded cohomology class. 
Lemma 7 is the last step in the proof of the following:

THEOREM 1. Assume that $G$ has trivial module action on abelian group $K$. There is a one-to-one correspondence between $\operatorname{Cent}_{b}(G, K)$ and $H_{b}^{2}(G, K)$.

3. Bounded cohomology spectral sequence. The construction of a spectral sequence demonstrates the usefulness of having a bounded condition on the cohomology groups. We follow the treatment in Mac Lane [2], and simply replace groups and homomorphisms with bounded groups and homomorphisms. Thus, a bounded cohomology spectral sequence $E=\left\{E_{r}, d_{r}\right\}$ is a sequence $E_{2}, E_{3}, \ldots$ of $\mathbf{Z}$-bigraded modules, each bounded as a group, and each with a bounded differential $d_{r}$ of bidegree $(r, 1-r)$ so that

$$
H\left(E_{r}, d_{r}\right)=E_{r+1}
$$

$H\left(E_{r}, d_{r}\right)$ has the quotient norm.

To demonstrate the potential usefulness of redefining the spectral sequence in this way, we construct the Lyndon spectral sequence. We can then relate the bounded cohomology of a group and its subgroup. Where inserting the word "bounded" in the standard arguments causes no problem we simply state the result. Complications arise later, however, and we deal with those in detail.

For $H$ a normal subgroup of bounded group $G$, and $A$ a bounded $G$-module, form the bicomplex

$$
K^{p, q}=\operatorname{Hom}_{G / H}^{b}\left(B_{p}(G / H), \operatorname{Hom}_{H}^{b}\left(B_{q}(G), A\right)\right) .
$$

The two differentials are defined, for $f$ in $K^{p, q}$,

$$
\begin{aligned}
& \delta^{\prime} f\left(b^{\prime}\right)\left(b^{\prime \prime}\right)=(-1)^{p+q+1} f\left(\partial b^{\prime}\right)\left(b^{\prime \prime}\right), \quad b^{\prime} \in B_{p+1}, b^{\prime \prime} \subset B_{q}, \\
& \delta^{\prime \prime} f\left(b^{\prime}\right)\left(b^{\prime \prime}\right)=(-1)^{q+1} f\left(b^{\prime}\right)\left(\partial b^{\prime \prime}\right), \quad b^{\prime} \in B_{p}, b^{\prime \prime} \in B_{q+1} .
\end{aligned}
$$

There are two filtrations of this bicomplex-by the indices $p$ and $q$. That each induces a bounded cohomology spectral sequence on Tot $K$ is laborious but straightforward. First we identify the $E_{2}$ terms.

Reverse the indices for the second filtration, and recall that $E_{2}^{\prime p, q}=$ $H^{\prime \prime} H^{\prime q}(K)$. For fixed $p, H^{\prime q}(K)$ is the bounded cohomology of $G / H$ with coefficients in $\operatorname{Hom}_{H}^{b}\left(B_{p}(G), A\right)$. This group $H^{\prime q}(K)$ is zero for $q>0 . H^{\prime 0}(K)$ is

$$
\begin{aligned}
H_{b}^{0}\left(G / H, \operatorname{Hom}_{H}^{b}\left(B_{p}(G), A\right)\right) & \cong_{b}\left[\operatorname{Hom}_{H}^{b}\left(B_{p}(G), A\right)\right]^{G / H} \\
& \cong_{b} \operatorname{Hom}_{G}^{b}\left(B_{p}(G), A\right) .
\end{aligned}
$$


Now calculating $H^{\prime \prime} H^{\prime q}(K)$ yields

$$
\begin{aligned}
& E_{2}^{\prime p, 0} \cong_{b} H_{b}^{p}(G, A), \\
& E_{2}^{\prime \prime, q}=0 \text { when } q>0 .
\end{aligned}
$$

The filtrations of $K$ determine the total space Tot $K$, a single complex, as

$$
\operatorname{Tot} K=\sum_{p+q=n} K^{p, q}, \quad \delta=\delta^{\prime}+\delta^{\prime \prime} .
$$

THEOREM 2. $H_{b}^{n}(G, A) \cong_{b} H_{b}^{n}(\operatorname{Tot} K)$.

Proof. Consider the following chain transformations:

$$
\begin{aligned}
& \zeta: \operatorname{Hom}_{G}^{b}(B(G), A) \rightarrow \operatorname{Tot} K, \\
& \eta: \operatorname{Tot} K \rightarrow \operatorname{Hom}_{G}^{b}(B(G), A) .
\end{aligned}
$$

For $f: B_{n}(G) \rightarrow A, \zeta f$ is an element in $K^{0, n}$ defined by

$$
(\zeta f)(u)(b)=f(b)
$$

for $u$ in $G / H, b$ in $B_{n}(G)$. The homomorphism $\zeta f$ has the same norm as $f$, and therefore $\zeta$ itself is a bounded chain transformation. For $h$ of degree $n$ in Tot $K, h=\left(h_{0}, \ldots, h_{n}\right)$ is an $(n+1)$-tuple of bounded homomorphisms, $h_{i}$ in $K^{i, n-i}$. Define $\eta h$, for $\left(x_{0}, \ldots, x_{n}\right)$ in $B_{0}(G)$,

$$
(\eta h)\left(x_{0}, \ldots, x_{n}\right)=\sum_{i=0}^{n}\left(h_{i}\left(\pi x_{0}, \ldots, \pi x_{i}\right)\right)\left(x_{i}, \ldots, x_{n}\right)
$$

The maps $\eta h$ and $\eta$ are easily seen to be bounded, and they satisfy the identity $\eta \zeta=1$. The following is a bounded chain homotopy between $\zeta$ and $\eta$.

Define $s_{n}: X^{n} \rightarrow X^{n-1}$, for $f$ in $X^{n}, u$ in $B_{p}(G / H)$, and $a$ in $B_{q}(G)$, $p+q=n$, by

$$
s_{n} f(u)(a)=(-1)^{q} \sum_{i=0}^{q} f\left(u_{0}, \ldots, u_{p}, \pi a_{0}, \ldots, \pi a_{i}\right)\left(a_{i}, \ldots, a_{q}\right) .
$$

Thus $\zeta$ and $\eta$ differ by the coboundary of a bounded $(n-1)$-cochain, $(\zeta \eta)^{*}=1$, and the cohomologies of the complexes are bounded isomorphic.

From the first spectral sequence, $E_{2}^{\prime p, q}=H^{\prime p} H^{\prime \prime q}(K)$. For fixed $p$,

$$
H^{\prime \prime q}(K)=\frac{\operatorname{Ker} \delta^{\prime \prime}: K^{p, q} \rightarrow K^{p, q+1}}{\operatorname{Im} \delta^{\prime \prime}: K^{p, q-1} \rightarrow K^{p, q}} .
$$

In the next result, restriction to the case when $G / H$ is finite allows us to obtain both the isomorphism and a bounded map. 
LEMMA 8. For $G / H$ finite,

$$
H^{\prime \prime q}(K) \cong_{b} \operatorname{Hom}_{G / H}^{b}\left(B_{p}(G / H), H_{b}^{q}\left(\operatorname{Hom}_{H}^{b}(B(G), A)\right)\right) .
$$

Proof. Given a class $x$ in $G^{\prime \prime q}(K)$, lift the class to $f_{p}$ in $K^{p, q}$ so that $\bar{f}_{p}=x$. Then send $x$ to $f: B_{p}(G / H) \rightarrow H_{b}^{q}\left(\operatorname{Hom}_{H}^{b}(B(G), A)\right)$, where $f(a)=\overline{f_{p}(a)}$. We will first show that this map is an isomorphism.

Suppose $f=0$. Then $f_{p}(a)$ is actually $\delta^{\prime \prime} h_{a}$, for $h_{a}$ in

$$
\operatorname{Hom}_{H}^{b}\left(B_{q-1}(G), A\right)
$$

and for all $a$. Now define $G$ in $K^{p, q-1}$ by

$$
G(a)=h_{a} .
$$

We know $G$ is bounded only because $G / H$ is finite, and thus

$$
\begin{gathered}
\operatorname{Hom}_{G / H}^{b}\left(B_{p}(G / H), V\right)=\operatorname{Hom}_{G / H}\left(B_{p}(G / H), V\right), \\
\delta^{\prime \prime} G(a)=\delta^{\prime \prime} h_{a}=f_{p}(a) .
\end{gathered}
$$

So $f_{p}$ is in the $\operatorname{Im} \delta^{\prime \prime}: K^{p, q-1} \rightarrow K^{p, q}$, and $\bar{f}_{p}=x=0$ in $H^{\prime \prime q}(K)$. The map is injective.

To show the map is onto, consider

$$
f: B_{p}(G / H) \rightarrow H_{b}^{q}\left(\operatorname{Hom}_{H}^{b}(B(G), A) .\right.
$$

Now define $f_{p}$ in $K^{p, q}$ by

$$
f_{p}(a)=s(f(a)),
$$

where $s$ is the bounded lifting for $H_{b}^{q}\left(\operatorname{Hom}_{H}^{b}(B(G), A)\right.$. The class $\bar{f}_{p}$ is sent to $f$, so the map is surjective.

The bounded nature of the isomorphism also depends on the finiteness of $G / H$. Let $L=\left\|\bar{f}_{p}\right\|$ in $H^{\prime \prime q}(K)$, and let $R=\|f\|$ on the right.

$$
\begin{gathered}
L=\left\|\bar{f}_{p}\right\|=\inf _{q \in K^{p, q-1}} \sup _{a \in B_{p}(G / H)}\left\|f_{p}(a)-\delta^{\prime \prime}(g(a))\right\|, \\
R=\sup _{a \in B_{p}(G / H)} \inf _{h \in \operatorname{Hom}_{H}^{b}\left(B_{q}, A\right)}\left\|f(a)-\delta^{\prime \prime} h\right\| .
\end{gathered}
$$

First we will demonstrate that $L \leq R$. For all $a$ in $B_{p}(G / H)$, $\inf _{\delta^{\prime \prime} h}\left\|f(a)-\delta^{\prime \prime} h\right\| \leq R$. So it is possible, given $\varepsilon>0$, for each $a$, to pick $h_{a}$ such that

$$
\left\|f(a)-\delta^{\prime \prime} h_{a}\right\| \leq R+\varepsilon
$$

Define $G$ in $K^{p, q-1}$ by

$$
G(a)=h_{a}
$$


Here we use the fact that $G / H$ is finite. Now look at the norm on the left.

$$
L=\inf _{g} \sup _{a}\left\|f(a)-\delta^{\prime \prime}(g(a))\right\| .
$$

The infinum over all $g$ is less than or equal to values obtained plugging in specific $g$ in $K^{p, q-1}$ :

$$
\begin{aligned}
L & \leq \sup _{a}\left\|f(a)-\delta^{\prime \prime}(G(a))\right\| \\
& =\sup _{a}\left\|f(a)-\delta^{\prime \prime} h_{a}\right\| \leq R+\varepsilon .
\end{aligned}
$$

This is true for all $\varepsilon$, so $L \leq R$.

To show $R \leq L$, we again start by considering the norm $R$. For each $\varepsilon>0$, pick $a_{\varepsilon}$ such that

$$
\begin{aligned}
& \inf _{\delta^{\prime \prime} h}\left\|f\left(a_{\varepsilon}\right)-\delta^{\prime \prime} h\right\| \geq R-\varepsilon . \\
& L=\inf _{g} \sup _{a}\left\|f(a)-\delta^{\prime \prime} g(a)\right\| .
\end{aligned}
$$

Fix $g$ and consider the $\sup _{a}\left\|f(a)-\delta^{\prime \prime} g(a)\right\|$. Each $g(a)$ is in $\operatorname{Hom}_{H}^{b}\left(B_{q-1}(G), A\right)$, and the $\sup _{a}$ includes $a_{\varepsilon}$. Therefore,

$$
\begin{gathered}
\sup _{a}\left\|f(a)-\delta^{\prime \prime} g(a)\right\| \geq\left\|f\left(a_{\varepsilon}\right)-\delta^{\prime \prime} g\left(a_{\varepsilon}\right)\right\| \\
\geq \inf _{\delta^{\prime \prime} h}\left\|f\left(a_{\varepsilon}\right)-\delta^{\prime \prime} h\right\| \geq R-\varepsilon .
\end{gathered}
$$

Since, for every $g, \sup _{a}\left\|f(a)-\delta^{\prime \prime} g(a)\right\| \geq R+\varepsilon$,

$$
L=\inf _{g} \sup _{a}\left\|f(a)-\delta^{\prime \prime} g(a)\right\| \geq R-\varepsilon .
$$

As a result, $L \geq R$; in fact we now know that $L=R$, so the isomorphism is bounded.

We state the following lemma without proof, for the isomorphism is generated by a standard chain transformation easily seen to be bounded.

LEMMA 9. $H_{b}^{n}\left(\operatorname{Hom}_{H}^{b}(B(G), A)\right) \cong_{b} H_{b}^{n}\left(\operatorname{Hom}_{n}^{b}(B(H), A)\right)$.

For $G / H$ finite, the previous lemmas allow us to assert the following:

$$
\begin{aligned}
H^{\prime \prime q}(K) & \cong_{b} \operatorname{Hom}_{G / H}\left(B_{p}(G / H), H_{b}^{q}\left(\operatorname{Hom}_{H}^{b}(B(G), A)\right)\right) \\
& \cong_{b} \operatorname{Hom}_{G / H}\left(B_{p}(G / H), H_{b}^{q}(H, A)\right) .
\end{aligned}
$$

Taking the cohomology of $H^{\prime \prime q, p}$ with respect to $\delta^{\prime}$ yields the cohomology of $G / H$ :

$$
E_{2}^{q, p} \cong_{b} H^{\prime p} H^{\prime \prime q} \cong_{b} H_{b}^{p}\left(G / H, H_{b}^{q}(H, A)\right) .
$$


Assume $A$ has characteristic zero. Because $G / H$ is finite, most of the terms of the spectral sequence vanish and a useful isomorphism emerges. The $E_{2}^{\prime p, q}$ term, for $p>0$ and $q \geq 0$ is the cohomology of a finite group with coefficients in a module of characteristic zero; thus the term is zero. The only non-trivial $E_{2}$ terms are on the fiber, and all differentials are zero. Therefore,

$$
E_{2}^{\prime 0, n}=E_{\infty}^{\prime 0, n}
$$

LEMMA $10 . E_{\infty}^{\prime 0, n} \cong_{b} H_{b}^{n}(\operatorname{Tot} K)$.

Proof. By definition,

$$
E_{\infty}^{\prime}=\frac{C^{n} \cup F^{1} X^{n}}{B^{n} \cup F^{1} x^{n}}, \quad H_{b}^{n}(\operatorname{Tot} K)=\frac{C^{n}}{B^{n}},
$$

where $C^{n}$ and $B^{n}$ are the cocycles and coboundaries of degree $n$. The isomorphism takes a class in $E_{\infty}^{\prime 0, n}$ to a representative $f+s, f$ in $C^{n}$ and $s$ in $F^{1} X^{n}$, then to $f$ in $C^{n} / B^{n}$. It remains to be shown that this isomorphism is bounded.

In $E_{\infty}^{\prime 0, n}$, the norm is calculated, for $f=f_{0} \oplus f_{1} \oplus \cdots \oplus f_{n}$,

$$
\begin{aligned}
L=\|f+s\|=\|\bar{f}\|=\inf _{\substack{\delta \in B^{n} \\
h \in F^{\prime} X^{n}}}\|f-\delta g-h\| & \\
=\inf _{\delta g, h}\left(\left\|f_{0}-\delta^{\prime \prime} g_{0}\right\|\right. & +\left\|f_{1}-\delta^{\prime} g_{0}-\delta^{\prime \prime} g_{1}-h_{1}\right\| \\
& \left.+\cdots+\left\|f_{n}-\delta^{\prime} g_{n-1}-h_{n}\right\|\right) .
\end{aligned}
$$

For any $\delta g$ we can choose $h$ so that $h_{i}=-\delta^{\prime} g_{i-1}-\delta^{\prime \prime} g_{i}$. Then,

$$
L=\inf _{\delta g_{0}}\left\|f-\delta^{\prime \prime} g_{0}\right\| \text {. }
$$

In $H_{b}^{n}($ Tot $K)$,

$$
R=\|\bar{f}\|=\inf _{\delta g \in B^{n}}\|f-\delta g\| .
$$

Now recall that $H^{p}\left(G / H, \operatorname{Hom}_{H}^{b}\left(B_{q}(H), A\right)\right)$ is zero when $p>0$. The homomorphism $f=f_{0} \oplus \cdots \oplus f_{n}$ is a cocycle, and thus $\delta^{\prime} f_{n}=0$. This implies that $f_{n}=\delta^{\prime} G_{n-1}$; since $\bar{f}=\overline{f-\delta G_{n-1}}$ we can replace $f$ by $F_{1}=f_{0} \oplus \cdots \oplus\left(f_{n-1}-\delta^{\prime \prime} G_{n-1}\right)$.

Now $F_{1}$ is a cocycle as well, so $f_{n-1}-\delta^{\prime \prime} G_{n-1}=\delta^{\prime} G_{n-2}$. Replace $F_{1}$ by $F_{2}=f_{0} \oplus \cdots \oplus\left(f_{n-2}-\delta^{\prime \prime} G_{n-2}\right)$. Repeat this procedure until left with

$$
\begin{aligned}
& F=f_{0}-\delta^{\prime \prime} G_{0}, \quad \text { where } \bar{F}=\bar{f} . \\
& R=\|\bar{f}\|=\|\bar{F}\|=\inf _{\delta^{\prime \prime} g}\left\|f_{0}-\delta^{\prime \prime} G_{0}-\delta^{\prime \prime} g\right\|=L .
\end{aligned}
$$


The isomorphism is bounded.

Combining this result with Theorem 2 yields

$$
H_{b}^{n}(H, A)^{G} \cong_{b} E_{\infty}^{\prime 0, n} \cong_{b} H_{b}^{n}(\operatorname{Tot} K) \cong_{b} H_{b}^{n}(G, A) .
$$

We have proved the following:

THEOREM 3. For $G / H$ finite, $H_{b}^{n}(H, A)^{G} \cong_{b} H_{b}^{n}(G, A)$.

\section{REFERENCES}

[1] Robert Brooks, Some Remarks on Bounded Cohomology, Proceedings of the Stony Brook Conference, (1978), 53-63.

[2] S. Mac Lane, Homology, Springer-Verlag, 1963.

[3] J. Milnor, Notes on Algebraic K-Theory, n.p., n.d.

[4] Calvin Moore, Extensions and low dimensional cohomology theory of locally compact groups, I and II, Annals of Math., October, 1964.

Received April 21, 1987.

FAIRLEIGH Dickenson University

MADISON, NJ 07940 


\section{EDITORS}

\author{
V. S. VARADARAJAN \\ (Managing Editor) \\ University of California \\ Los Angeles, CA 90024 \\ HeRBert Clemens \\ University of Utah \\ Salt Lake City, UT 84112 \\ R. FINN \\ Stanford University \\ Stanford, CA 94305
}

\author{
HERMANN FLASCHKA \\ University of Arizona \\ Tucson, AZ 85721
}

RAMESh A. GANGOLLI University of Washington Seattle, WA 98195

VAUGHAN F. R. JONES University of California

Berkeley, CA 94720
ROBION KIRBY

University of California

Berkeley, CA 94720

C. C. MOORE

University of California

Berkeley, CA 94720

HAROLD STARK

University of California, San Diego

La Jolla, CA 92093

\section{ASSOCIATE EDITORS}
R. ARENS
E. F. BECKENBACH
B. H. NEUMANN
F. WOLF
K. YOSHIDA

(1906-1982)

\section{SUPPORTING INSTITUTIONS}

UNIVERSITY OF ARIZONA
UNIVERSITY OF BRITISH COLUMBIA
CALIFORNIA INSTITUTE OF TECHNOLOGY
UNIVERSITY OF CALIFORNIA
MONTANA STATE UNIVERSITY
UNIVERSITY OF NEVADA, RENO
NEW MEXICO STATE UNIVERSITY
OREGON STATE UNIVERSITY

UNIVERSITY OF ARIZONA

UNIVERSITY OF BRITISH COLUMBIA

UNIVERSITY OF CALIFORNIA

MONTANA STATE UNIVERSITY

NEW MEXICO STATE UNIVERSITY

OREGON STATE UNIVERSITY

\author{
UNIVERSITY OF OREGON \\ UNIVERSITY OF SOUTHERN CALIFORNIA \\ STANFORD UNIVERSITY \\ UNIVERSITY OF HAWAII \\ UNIVERSITY OF TOKYO \\ UNIVERSITY OF UTAH \\ WASHINGTON STATE UNIVERSITY \\ UNIVERSITY OF WASHINGTON
}

The Supporting Institutions listed above contribute to the cost of publication of this Journal, but they are not owners or publishers and have no responsibility for its content or policies.

Mathematical papers intended for publication in the Pacific Journal of Mathematics should be in typed form or offset-reproduced (not dittoed), double spaced with large margins. Please do not use built up fractions in the text of the manuscript. However, you may use them in the displayed equations. Underline Greek letters in red, German in green, and script in blue. The first paragraph must be capable of being used separately as a synopsis of the entire paper. In particular it should contain no bibliographic references. Please propose a heading for the odd numbered pages of less than 35 characters. Manuscripts, in triplicate, may be sent to any one of the editors. Please classify according to the scheme of Math. Reviews, Index to Vol. 39. Supply name and address of author to whom proofs should be sent. All other communications should be addressed to the managing editor, or Elaine Barth, University of California, Los Angeles, California 90024.

There are page-charges associated with articles appearing in the Pacific Journal of Mathematics. These charges are expected to be paid by the author's University, Government Agency or Company. If the author or authors do not have access to such Institutional support these charges are waived. Single authors will receive 50 free reprints; joint authors will receive a total of 100 free reprints. Additional copies may be obtained at cost in multiples of 50 .

The Pacific Journal of Mathematics is issued monthly as of January 1966. Regular subscription rate: $\$ 190.00$ a year (5 Vols., 10 issues). Special rate: $\$ 95.00$ a year to individual members of supporting institutions.

Subscriptions, orders for numbers issued in the last three calendar years, and changes of address should be sent to Pacific Journal of Mathematics, P.O. Box 969, Carmel Valley, CA 93924, U.S.A. Old back numbers obtainable from Kraus Periodicals Co., Route 100, Millwood, NY 10546.

The Pacific Journal of Mathematics at P.O. Box 969, Carmel Valley, CA 93924 (ISSN 0030-8730) publishes 5 volumes per year. Application to mail at Second-class postage rates is pending at Carmel Valley, California, and additional mailing offices. Postmaster: send address changes to Pacific Journal of Mathematics, P.O. Box 969, Carmel Valley, CA 93924.

\section{PUBLISHED BY PACIFIC JOURNAL OF MATHEMATICS, A NON-PROFIT CORPORATION} Copyright (c) 1988 by Pacific Journal of Mathematics 


\section{Pacific Journal of Mathematics}

Vol. 134, No. $2 \quad$ June, 1988

P. D. Allenby and M. Sears, Extension of flows via discontinuous functions

Arthur William Apter and Moti Gitik, Some results on Specker's

problem

Shiu-Yuen Cheng and Johan Tysk, An index characterization of the catenoid and index bounds for minimal surfaces in $\mathbf{R}^{4}$

Mikihiro Hayashi and Mitsuru Nakai, Point separation by bounded analytic functions of a covering Riemann surface

Charles Philip Lanski, Differential identities, Lie ideals, and Posner's theorems

Erich Miersemann, Asymptotic expansion at a corner for the capillary problem

Dietrich W. Paul, Theory of bounded groups and their bounded cohomology

Ibrahim Salama, Topological entropy and recurrence of countable chains

Zbigniew Slodkowski, Pseudoconvex classes of functions. I. Pseudoconcave

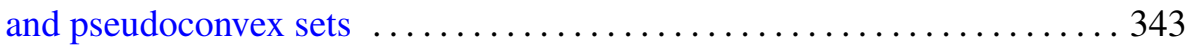

Alfons Van Daele, $K$-theory for graded Banach algebras. II . . . . . . . . . . 377

Edwin Wolf, Functions in $R^{2}(E)$ and points of the fine interior 393 